\title{
Functional Polymorphisms in the TERT Promoter Are Associated with Risk of Serous Epithelial Ovarian and Breast Cancers
}

Jonathan Beesley ${ }^{1 * 9}$, Hilda A. Pickett' ${ }^{2,39}$, Sharon E. Johnatty ${ }^{1}$, Alison M. Dunning ${ }^{4}$, Xiaoqing Chen ${ }^{1}$, Jun Li $^{1}$, Kyriaki Michailidou ${ }^{4}$, Yi Lu' ${ }^{1}$, David N. Rider ${ }^{5}$, Rachel T. Palmieri', Michael D. Stutz ${ }^{2,3}$, Diether Lambrechts $^{7,8}$, Evelyn Despierre ${ }^{9}$, Sandrina Lambrechts ${ }^{9}$, Ignace Vergote ${ }^{9}$, Jenny Chang-Claude ${ }^{10}$, Stefan Nickels $^{10}$, Alina Vrieling ${ }^{10}$, Dieter Flesch-Janys ${ }^{11}$, Shan Wang-Gohrke ${ }^{12}$, Ursula Eilber ${ }^{10}$, Natalia Bogdanova $^{13,14,15}$, Natalia Antonenkova ${ }^{14}$, Ingo B. Runnebaum ${ }^{16}$, Thilo Dörk ${ }^{13}$, Marc T. Goodman ${ }^{17}$, Galina Lurie ${ }^{17}$, Lynne R. Wilkens ${ }^{17}$, Rayna K. Matsuno ${ }^{17}$, Lambertus A. Kiemeney ${ }^{18,19,20}$, Katja K. H. Aben $^{18,20}$, Tamara Marees ${ }^{18}$, Leon F. A. G. Massuger ${ }^{21}$, Brooke L. Fridley ${ }^{5}$, Robert A. Vierkant ${ }^{5}$, Elisa V. Bandera $^{22}$, Sara H. Olson ${ }^{23}$, Irene Orlow ${ }^{23}$, Lorna Rodriguez-Rodriguez ${ }^{22}$, Linda S. Cook ${ }^{24}$, Nhu D. Le ${ }^{24}$, Angela Brooks-Wilson ${ }^{24}$, Linda E. Kelemen ${ }^{24}$, lan Campbell ${ }^{25}$, Simon A. Gayther ${ }^{26}$, Susan J. Ramus ${ }^{26}$, Aleksandra Gentry-Maharaj ${ }^{27}$, Usha Menon ${ }^{27}$, Shahana Ahmed ${ }^{4}$, Caroline Baynes ${ }^{4}$, Paul D. Pharoah ${ }^{4}$, kConFab Investigators ${ }^{25}$, Australian Ovarian Cancer Study Group ${ }^{1,25,28}$, ABCTB Investigators, Kenneth Muir $^{30}$, Artitaya Lophatananon ${ }^{30}$, Arkom Chaiwerawattana ${ }^{31}$, Surapon Wiangnon ${ }^{32}$, Stuart Macgregor ${ }^{1}$, Douglas F. Easton ${ }^{4}$, Roger R. Reddel ${ }^{2,3}$, Ellen L. Goode ${ }^{5}$, Georgia Chenevix-Trench ${ }^{1}$ on behalf of the Ovarian Cancer Association Consortium

1 Division of Genetics and Population Health, Queensland Institute of Medical Research, Brisbane, Queensland, Australia, 2 Cancer Research Unit, Children's Medical Research Institute, Westmead, New South Wales, Australia, 3 Sydney Medical School, University of Sydney, Sydney, New South Wales, Australia, 4 Department of Oncology, University of Cambridge, Strangeways Research Laboratory, Cambridge, United Kingdom, 5 Department of Health Sciences Research, Mayo Clinic College of Medicine, Rochester, Minnesota, United States of America, $\mathbf{6}$ Department of Community and Family Medicine, Duke University Medical Center, Durham, North Carolina, United States of America, $\mathbf{7}$ Vesalius Research Center, VIB, Leuven, Belgium, $\mathbf{8}$ Vesalius Research Center, University of Leuven, Leuven, Belgium, 9 Division of Gynaecologic Oncology, Department of Obstetrics and Gynaecology, University Hospitals Leuven, University of Leuven, Leuven, Belgium, 10 Division of Cancer Epidemiology, German Cancer Research Center (DKFZ), Heidelberg, Germany, 11 Institute for Medical Biometrics and Epidemiology, University Clinic Hamburg-Eppendorf, Hamburg, Germany, 12 Department of Obstetrics and Gynecology, University of Ulm, Ulm, Germany, 13 Clinics of Obstetrics and Gynaecology, Hannover Medical School, Hannover, Germany, 14 Byelorussian Institute for Oncology and Medical Radiology Aleksandrov N.N., Minsk, Belarus, 15 Clinics of Radiation Oncology, Hannover Medical School, Hannover, Germany, 16 Clinics of Obstetrics and Gynaecology, Friedrich Schiller University, Jena, Germany, 17 Cancer Epidemiology Program, University of Hawaii Cancer Center, Honolulu, Hawaii, United States of America, 18 Department of Epidemiology, Biostatistics and HTA, Radboud University Nijmegen Medical Centre, Nijmegen, The Netherlands, 19 Department of Urology, Radboud University Nijmegen Medical Centre, Nijmegen, The Netherlands, $\mathbf{2 0}$ Comprehensive Cancer Center, The Netherlands, Nijmegen, The Netherlands, 21 Department of Gynaecology, Radboud University Nijmegen Medical Centre, Nijmegen, The Netherlands, 22 The Cancer Institute of New Jersey/Robert Wood Johnson Medical School, New Brunswick, New Jersey, United States of America, 23 Department of Epidemiology \& Biostatistics, Memorial Sloan Kettering Cancer Center, New York, New York, United States of America, 24 Department of Population Health Research, Alberta Health Services-Cancer Care, Calgary, Alberta, Canada, 25 Peter MacCallum Cancer Centre, East Melbourne, Victoria, Australia, 26 Department of Preventive Medicine, Keck School of Medicine, University of Southern California, Los Angeles, California, United States of America, 27 Department of Gynaecological Oncology, University College London, EGA Institute for Women's Health, London, United Kingdom, 28 Department of Gynaecological Oncology and Westmead Institute for Cancer Research, University of Sydney at the Westmead Millennium Institute, Westmead Hospital, Sydney, New South Wales, Australia, 29 Westmead Millennium Institute, Sydney Medical School, Westmead, The University of Sydney, Sydney, New South Wales, Australia, 30 Health Sciences Research Institute, Warwick Medical School, University of Warwick, Coventry, United Kingdom, 31 Department of Academic Support, The National Cancer Institute of Thailand, Ministry of Public Health, Nonthaburi, Thailand, 32 Department of Pediatrics, Medical School, Khon Kaen University, Khon Kaen, Thailand

\section{Abstract}

Genetic variation at the TERT-CLPTM1L locus at $5 p 15.33$ is associated with susceptibility to several cancers, including epithelial ovarian cancer (EOC). We have carried out fine-mapping of this region in EOC which implicates an association with a single nucleotide polymorphism (SNP) within the TERT promoter. We demonstrate that the minor alleles at rs2736109, and at an additional TERT promoter SNP, rs2736108, are associated with decreased breast cancer risk, and that the combination of both SNPs substantially reduces TERT promoter activity. 
Citation: Beesley J, Pickett HA, Johnatty SE, Dunning AM, Chen X, et al. (2011) Functional Polymorphisms in the TERT Promoter Are Associated with Risk of Serous Epithelial Ovarian and Breast Cancers. PLoS ONE 6(9): e24987. doi:10.1371/journal.pone.0024987

Editor: Lin Zhang, University of Pennsylvania School of Medicine, United States of America

Received August 12, 2011; Accepted August 21, 2011; Published September 15, 2011

This is an open-access article distributed under the terms of the Creative Commons Public Domain declaration which stipulates that, once placed in the public domain, this work may be freely reproduced, distributed, transmitted, modified, built upon, or otherwise used by anyone for any lawful purpose.

Funding: The Ovarian Cancer Association Consortium is supported by a grant from the Ovarian Cancer Research Fund. kConFaB is supported by the National Breast Cancer Foundation, the National Health and Medical Research Council of Australia, and the Cancer Councils of Oueensland, New South Wales, Western Australia, South Australia, and Victoria. The Clinical Follow-up Study of kConFaB is funded by the National Health and Medical Research Council (NHMRC) of Australia (145684 and 288704). AOCS was supported by the U.S. Army Medical Research and Materiel Command (DAMD17-01-1-0729), the National Health and Medical Research Council of Australia (199600), and the Cancer Council Tasmania and Cancer Foundation of Western Australia. Tissues and samples were received from the Australian Breast Cancer Tissue Bank which is supported by the National Health and Medical Research Council of Australia, The Cancer Institute NSW and the National Breast Cancer Foundation. The tissues and samples are made available to researchers on a non-exclusive basis. The BEL study was supported by the Nationaal Kankerplan - actie 29. The HAW study is supported by the U.S. National Institutes of Health (R01 CA58598, N01-CN-55424, N01-PC-67001). SEARCH was supported by Cancer Research-UK grants [C1287/A10118, C490/A11021, C8197/A10123]. The MAYO study was supported by R01 CA122443 and P50 CA136939. The MARIE study was supported by the Deutsche Krebshilfe e.V., grant number 70-2892-BR I, the Hamburg Cancer Society, the German Cancer Research Center (DKFZ) and the DNA extraction and genotype work in part by the Federal Ministry of Education and Research (BMBF) Germany grant 01KH0402. The GESBC epidemiological study was supported by the Deutsche Krebshilfe e. V. [70492] and GESBC genotyping in part by the state of Baden-Württemberg through the Medical Faculty of the University of UIm [P.685]. The OVAL-BC Study is supported by the British Columbia Workers Compensation Board and the Canadian Institutes for Health Research. The NJO study was funded by NIH-K07 CA095666, R01CA83918, and The Cancer Institute of New Jersey. The UKO study is supported by funding from Cancer Research UK, the Eve Appeal, and the OAK Foundation; some of this work was undertaken at UCLH/UCL which received some funding from the Department of Health's NIHR Biomedical Research Centre funding scheme. ACP study is funded by The Breast Cancer Research Trust, UK. JB, HAP, SEJ, MDS and RRR are supported by NHMRC project grant 1012023. DFE is a Principal Research Fellow of Cancer Research UK. SM is supported by an NHMRC career development award. The funders had no role in study design, data collection and analysis, decision to publish, or preparation of the manuscript.

Competing Interests: The authors have declared that no competing interests exist.

* E-mail: jonathan.beesley@qimr.edu.au

9 These authors contributed equally to this work.

\section{Introduction}

Genome-wide association studies (GWAS) have identified more than 140 cancer susceptibility loci for 17 different cancers (www. genome.gov/gwastudies), including a locus at 5p15.33 which has been implicated in susceptibility to melanoma [1,2], glioma [3], lung [4,5], pancreas [6], prostate [2], testicular [7], and bladder cancers [8]. This locus harbours TERT, encoding the reverse transcriptase component of telomerase, and cleft lip and palate transmembrane protein 1-like (CLPTM1L). GWAS of ovarian and breast cancer have not detected an association with this locus to date, which may be due to poor tagging of this region on the chips employed, or the lack of statistical power to detect associations. Using a candidate gene approach we previously reported evidence of an association between an intronic SNP in TERT (rs7726159) and EOC risk, particularly of the serous histological subtype, using a per-allele model [9]. In this study we carried out fine-mapping, followed by functional analyses of associated SNPs identified within the promoter region of TERT. We directly demonstrate that the presence of a common haplotype, which is associated with decreased cancer risk, substantially reduces TERT promoter activity. The vast majority of cancers depend on expression of telomerase, which requires substantial upregulation of TERT expression, for their continued proliferation, strongly implicating TERT as the predominant gene involved in this association.

\section{Results and Discussion}

To further clarify the association previously reported with TERT, we employed a fine-mapping strategy in nine case-control studies from the Ovarian Cancer Association Consortium (OCAC) (Table S1). SNPs in a region spanning $\pm 250 \mathrm{~kb}$ across the TERT, CLPT1ML, SLC6A18 and SLC6A19 genes that were correlated $\left(0.2 \leq \mathrm{r}^{2} \leq 0.99\right)$ with $\mathrm{rs} 7726159$, rs11133719, rs2735940 and rs2736100 [9] were selected from the 1000 Genomes low coverage pilot release of April 2009. The SNPs implicated by our previous study of EOC [9] (rs11133719, rs7726159, rs2736100 and rs2735940) or by cancer GWAS (rs2736100) were also included in the panel. We genotyped 36 SNPs by iPLEX (Sequenom Inc.) in 2,130 invasive EOC cases and 3,975 controls, all of Caucasian ancestry. After excluding monomorphic loci $(n=6)$ and two SNPs that failed OCAC's quality control criteria [10], 28 SNPs were analysed for association with risk of EOC. We used single marker and stepwise logistic regression models, adjusted for study and age (at interview for controls and at diagnosis for cases) with a threshold of $P \leq 0.05$ for addition (forward stepwise) or removal (backward stepwise) of SNPs (Table S2). We found that rs2736109 showed the strongest association with serous EOC (adjusted $\left.\mathrm{OR}_{\text {per-allele }} 0.86(0.77-0.96), P=0.005\right)$ (Table S3), but not with invasive EOG risk overall (adjusted $\mathrm{OR}_{\text {per-allele }} 0.96$ (0.89-1.05), $P=0.38$ ) (data not shown). Likelihood ratio tests comparing logistic regression models with and without a genotypeby-study interaction term revealed no significant study heterogeneity $(P=0.4)$. rs2736109 is in a region of low linkage disequilibrium that encompasses the $5^{\prime}$ end of the TERT gene and the TERT promoter (Figure 1). This region also contains the SNPs, rs2736108 and rs2853669, which have pairwise correlations $\left(r^{2}\right)$ with each other, and with rs2736109 of greater than 0.6. It has previously been reported that $\mathrm{rs} 2853669$ is associated with breast cancer risk [11].

Since other loci confer susceptibility to both EOG and breast cancer [12,13] we investigated associations of TERT SNPs with breast cancer risk in various data sets. First, we genotyped rs2736109 by iPLEX in 4,277 invasive breast cancer cases and 7,000 controls from Australian, German and Thai studies from the Breast Cancer Association Consortium (BCAC) (Table S4). In the combined analysis of the Australian and German studies, there was no association with invasive breast cancer risk overall (adjusted $\left.\mathrm{OR}_{\text {per-allele }} 0.95(0.90-1.01), P=0.10\right)$, but there was an association in cases $\geq 50$ years at diagnosis that approached significance (adjusted $\left.\mathrm{OR}_{\text {per-allele }} 0.94(0.88-1.00), P=0.049\right)$. We also found a stronger association for ER-negative tumours (adjusted $\mathrm{OR}_{\text {per-allele }} 0.88(0.80-0.98), P=0.022$; for ER-positive tumours adjusted $\left.\mathrm{OR}_{\text {per-allele }} 0.98(0.92-1.05), P=0.562\right)$ (Tables S5, S6, and S7). We found the strongest evidence of association among ER-negative cases over the age of $50(n=636)$ (adjusted $\left.\mathrm{OR}_{\text {per-allele }} 0.84(0.75-0.95), P=0.005\right)$. We did not find any 


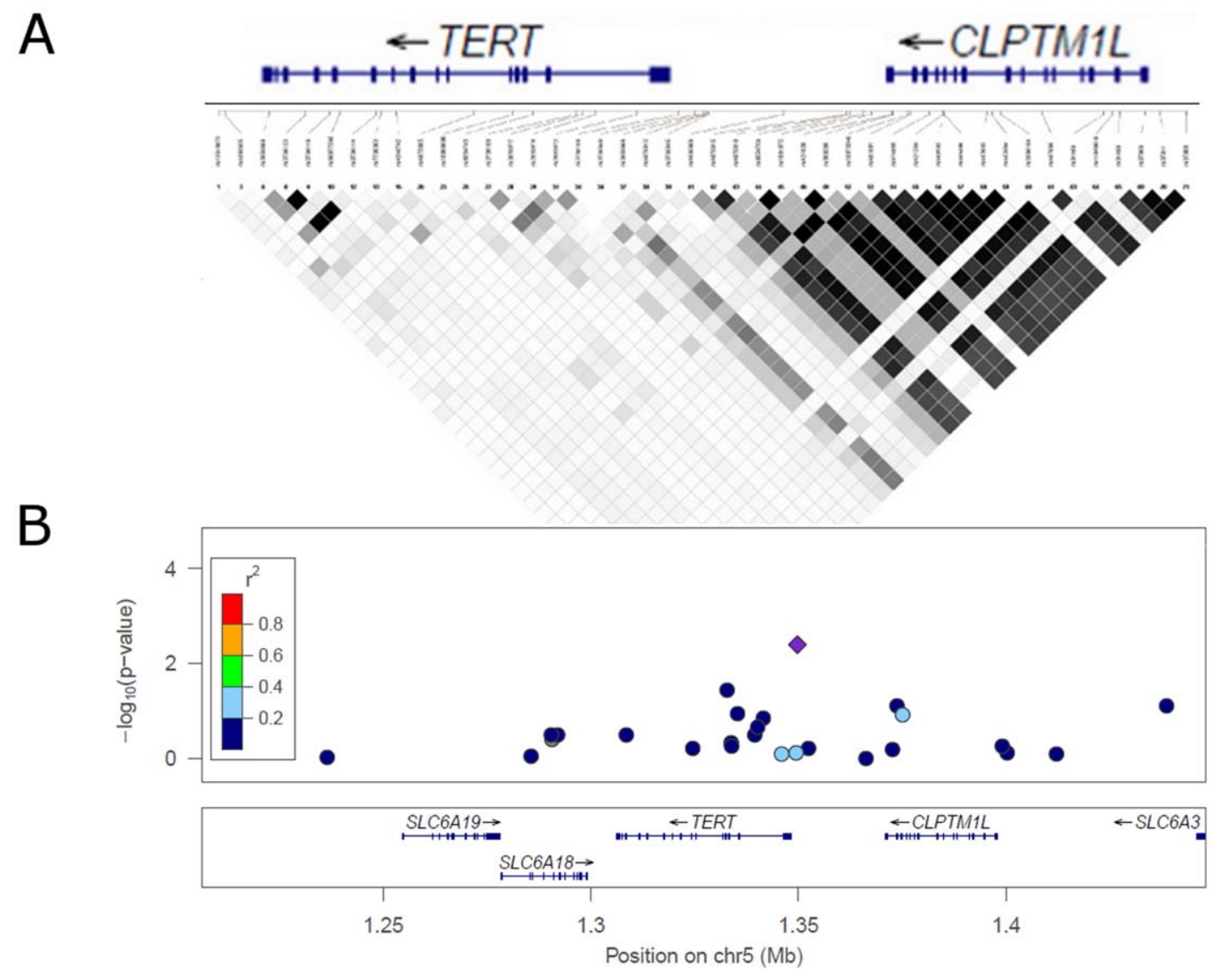

Figure 1. TERT-CLPTM1L locus SNPs genotyped in epithelial ovarian cancer cases and controls. Panel $\mathbf{A}$ depicts the pattern of linkage disequilibrium using data from the HapMap phase II+III CEU population (2010), where white represents $r^{2}=0$, and black $r^{2}=1$. The plot in $\mathbf{B}$ represents $-\log _{10} p$ against the chromosomal position for genotyped SNPs in the ovarian cancer fine mapping panel. The purple diamond represents the SNP (rs2736109) with the strongest observed association. The point colours represent the strength of LD according to 1000 Genomes Data. doi:10.1371/journal.pone.0024987.g001

association with breast cancer risk in a Thai study ( $\mathrm{n}=327$ cases) genotyped for rs2736109.

Next, we analysed genotypes imputed using $\mathrm{MaCH}$ for rs2736109 $\left(\mathrm{r}^{2}=0.45\right)$ from a breast cancer GWAS of 3,931 cases and 3,622 controls from the United Kingdom, from which neither age nor ER status were available [14]. We observed a significant association between rs2736109 and overall breast cancer risk in this population $\left(\mathrm{OR}_{\text {per-allele }} 0.91(0.83-0.99), P=0.037\right)$, and from a weighted meta-analysis of imputed and genotyped data from all studies $\left(\mathrm{OR}_{\text {per-allele }} 0.94(0.89-0.98), P=0.011\right)$.

In an additional replication sample set (SEARCH) we genotyped a correlated SNP, rs2736108 $\left(\mathrm{r}^{2}=0.96,1000\right.$ Genomes Project, Dec 2009, $\mathrm{r}^{2}=0.63$ based on 345 Australian controls) in 6,788 cases and 6,426 controls because rs2736109 was not amenable to genotyping by TaqMan (the genotyping platform used by this group). We observed a significant association with breast cancer risk overall (adjusted $\mathrm{OR}_{\text {per-allele }} 0.92$ (0.87-0.97), $P=0.003)$, which was restricted to the subset of cases diagnosed at age 50 or older (adjusted $\mathrm{OR}_{\text {per-allele }} 0.91(0.85-0.97), P=0.002$ ) (Table S6). Estimates according to ER status showed a significant association between the rs2736108 genotypes and ER-positive tumours (adjusted $\mathrm{OR}_{\text {per-allele }} 0.93(0.88-0.99), P=0.031$ ); there was no significant association in ER-negative tumours but the estimated OR was similar $\left(\mathrm{OR}_{\text {per-allele }} 0.95(0.85-1.06)\right)$ and the sample size much smaller. Comparison of models for both rs2736109 and rs2736108 with and without genotype by age group interaction terms showed no evidence of a statistical interaction on the multiplicative scale $\left(P_{\text {interaction }} \geq 0.25\right)$.

The SNPs of interest (rs2736108, rs2736109 and rs2853669) lie within the upstream promoter region of TERT. To determine the functional significance of these sites, we generated combinations of these variants in a luciferase reporter construct containing $3.9 \mathrm{~kb}$ of the TERT promoter [15]. Relative promoter activity was determined in an EOC cell line (27/87), a breast adenocarcinoma cell line (MDA-MB-468), and in post-selection normal breast epithelial cells (Bre16) (Figure 2). Introduction of a mutation into the human estrogen-responsive element in the TERT promoter (TERT-ERE) [16] was used as a positive control and confirmed diminished reporter activity. In all three cell types, luciferase activity was substantially reduced for the construct carrying both minor (A) alleles at rs2736108 and rs2736109, but remained unaltered for those with the individual minor allele at either SNP. We observed no change in expression for the minor allele at rs2853669.

Our analysis of Australian controls estimated a frequency of $32 \%$ for the A-A haplotype at rs2736108 and rs2736109, suggesting that this relatively common promoter haplotype may lower the risk of ovarian and breast cancer through decreasing TERT expression. This finding provides no support for the hypothesis that decreased telomerase activity predisposes to genomic instability and consequent oncogenic progression but instead our data imply the opposite, namely, that decreased TERT expression confers decreased cancer risk. 
A

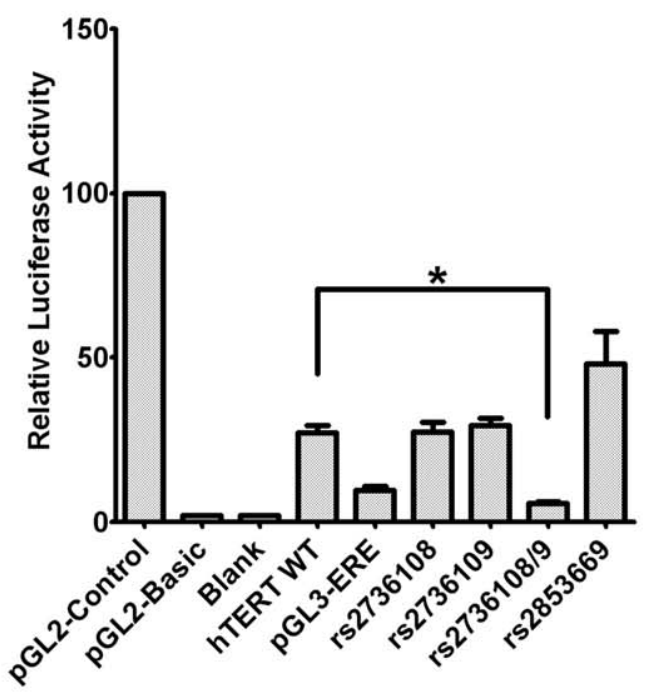

B
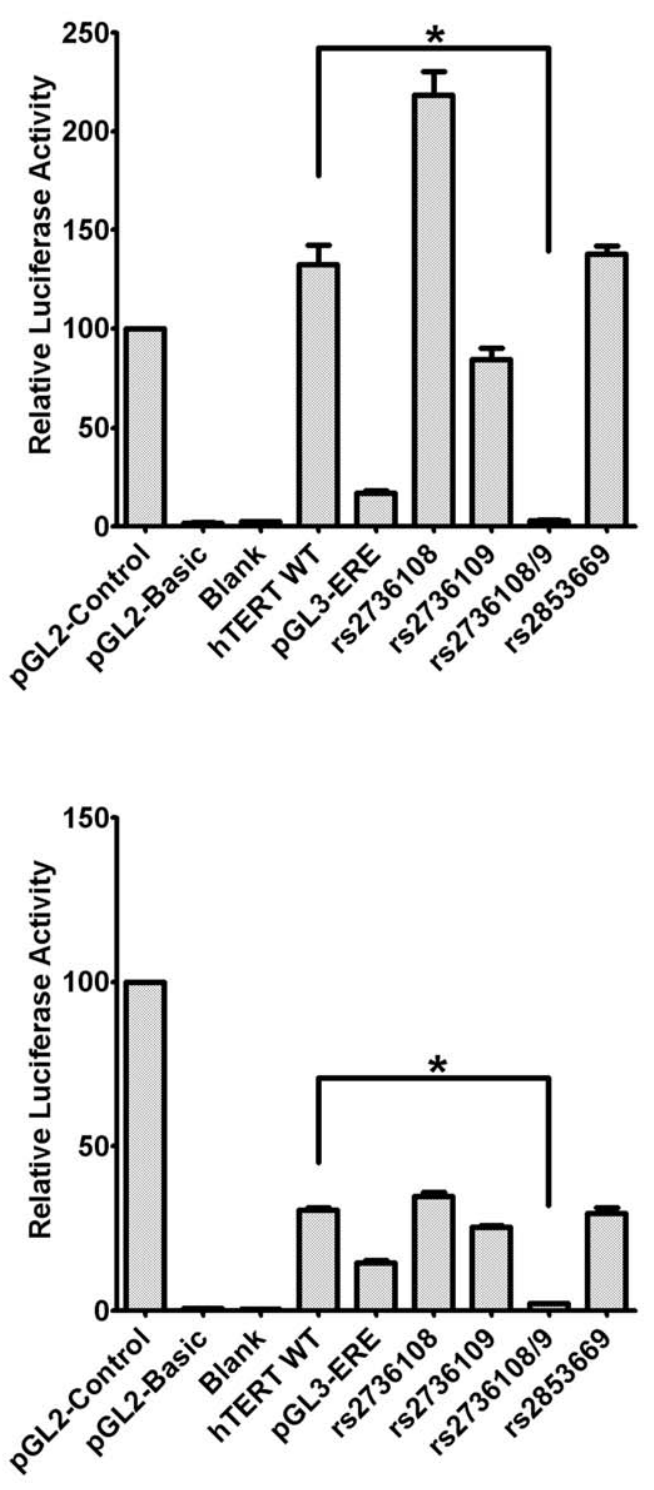

Figure 2. TERT promoter activity. Luciferase reporter assays following transient transfection with pGL2-control (SV40 promoter and enhancer), pGL2-basic (lacks promoter and enhancer), transfection control (blank), and the TERT reporter vectors TERT WT (3.9 kb of TERT promoter), the positive control TERT-ERE (containing a mutation in the estrogen-responsive element), and the minor alleles of rs2736108 (A allele), rs2736109 (A allele), rs2736108/9 (A alleles at both sites), rs2853669 (C allele) in (A) an EOC cell line (27/87), (B) a breast adenocarcinoma cell line (MDA-MB-468) and (C) a normal breast epithelial cell strain (Bre16). Error bars represent standard deviation between three separate experiments. ${ }^{*}$ represents statistical significance using one-way ANOVA with post hoc Dunnett's tests. doi:10.1371/journal.pone.0024987.g002

We also examined tumour expression and germline variants at the TERT-CLPTM1L locus using data from The Cancer Genome Atlas (TCGA) ovarian serous cystadenocarcinoma set. mRNA expression profiling was available for 574 tumour samples using Affymetrix U133A platform This assay has one probe for TERT, but none for CLPTM1L. TERT was expressed at low levels, as expected, but this does not necessarily reflect low levels of telomerase protein expression or activity [17]. 508 normal DNA samples were genotyped using Illumina $1 \mathrm{M}$ array, and the SNP rs2736108 was typed on this array. We also imputed the TCGA samples with reference to the 1000 Genome data (June 2010 release); rs2853669 was successfully imputed, but rs2736109 failed quality control. There was no evidence suggesting that the genotyped SNP, rs2736108, or the imputed SNP, rs2853669, are associated with expression of TERT. However one SNP, rs2735845, in partial linkage disequilibrium with the typed SNP, rs2736108 $\left(r^{2}=0.306\right)$, was associated with significantly altered transcript abundance of TERT $\left(P=4 \times 10^{-4}\right.$ and $P=0.0029$ after correction for multiple testing). Gene copy number aberration was found in TERT; it was amplified in $\sim 20 \%$ of tumour samples. This SNP showed robust association after adjusting for the copy number variation $\left(P=1.8 \times 10^{-3}\right.$ and $P=0.015$ after correction for multiple testing), and it explained $1.85 \%$ of variance of TERT transcription in ovarian cancer tumours.

A recently published meta-analysis identified a significantly decreased risk of breast cancer with a TERT SNP, rs2853669 (OR $0 \cdot 76(0 \cdot 64-0 \cdot 91), P=0 \cdot 002)[11]$. This SNP lies in the same linkage disequilibrium block as rs2736108 and rs2736109 $\left(\mathrm{r}^{2}>0.6\right.$ with rs2736108); therefore, this meta-analysis provides additional support for an association between TERT promoter SNPs and breast cancer risk. However, our functional analyses, which were carried out in normal breast epithelial cells and a breast adenocarcinoma cell line did not indicate any change to TERT expression with this individual SNP.

We computed a gene-based test [18] of association at TERT and CLPTM1L which yielded evidence in GWAS data available from dbGAP (http://www.ncbi.nlm.nih.gov/gap) for association with risk of lung, prostate and pancreatic cancer, but not breast cancer overall. Combining data for all cancers in a cross-cancer meta-analysis, revealed a genome-wide significant gene-based $P=4.1 \times 10^{-7}$ for CLPTM1L $(P=0.008$ after correction for 19,000 genes). A similar result was obtained for TERT (all cancer $\left.P=7.7 \times 10^{-5}\right)$. This gene-based test includes all SNPs within $50 \mathrm{~kb}$ of the start/stop site of each gene. Most of the associated SNPs lie in the interval between TERT and CLPTM1L but with slightly more evidence for SNPs near to CLPTM1L, leading to a slightly higher gene-based $P$ value for CLPTM1L. These results are based on marker data from Illumina GWAS arrays and hence the exact location of the maximum association test statistic is dependent to some degree upon the arbitrary set of SNPs that are on the arrays. Clearly, additional analysis of the entire TERT- 
CLPM1L locus is warranted in breast, and other, cancers to validate the associations we have identified and fine map putative causal variants if our findings are confirmed.

The failure to date to identify an association between the TERT-CLPTM1L locus and risk of EOC or breast cancer by GWAS may be explained by the pattern of linkage disequilibrium of the relevant SNPs: rs2736100, the tagSNP most commonly identified by GWAS of other cancers is poorly correlated with rs2736108 and rs2736109 $\left(\mathrm{r}^{2}=0.141\right.$ and 0.105 , respectively), and neither of these SNPs are on the Illumina $300 \mathrm{~K}, 610 \mathrm{~K}$ or $650 \mathrm{~K}$ arrays used for most cancer GWAS.

In summary, we have demonstrated a direct association between functional SNPs in the TERT promoter, which confer decreased risk of ovarian and breast cancer, and reduced TERT promoter activity. Decreased levels of TERT result in progressive telomere shortening and the onset of cellular senescence, which ultimately acts to suppress tumorigenesis. The association of hypomorphic sequence variants in the TERT promoter with decreased risk of cancer implicates downregulation of telomerase and telomere shortening as an intrinsic tumour suppressive mechanism. It is also possible that TERT variants associated with elevated cancer risk may alter the stringency with which TERT is regulated, potentially facilitating TERT activation and consequently providing a tumorigenic advantage. Potential noncanonical roles of TERT in cell signalling pathways may also underlie cancer risk [19]. Our results add functional insight into the increasingly important role of TERT as a cancer risk factor and demonstrate the need for further mechanistic analysis of this multicancer susceptibility locus.

\section{Materials and Methods}

\section{Ethics Statement}

Approval for this study was obtained from The Queensland Institute of Medical Research Human Research Ethics Committee. All studies were approved by the review boards and ethics committees of their respective institutions, and all participants provided written informed consent.

\section{Genotyping}

iPLEX genotyping was carried out using MALDI-TOF spectroscopy utilising Sequenom's MassARRAY platform and iPLEX GOLD chemistry. $10 \mathrm{ng}$ of genomic DNA was used as template, to which a PCR mix containing Qiagen Hot-StarTaq was added. Shrimp alkaline phosphatase and primer extension steps were carried out using Sequenom's protocol and reagents. Primers were obtained from Integrated DNA Technologies (Ohio USA). Assays were designed with MassARRAY Assay Design version 3.1(Sequenom). Raw genotype data were visualised and processed with MassARRAY Typer software version 3.4. TaqMan genotyping (SEARCH) was carried out as previously described [20]. Strict quality control criteria were adhered to as part of the Ovarian Cancer Association Consortium's guidelines [10].

\section{Statistical methods}

We used single marker and stepwise logistic regression models to screen $28 \mathrm{SNPs}$ in non-Hispanic white ovarian cancer cases $(\mathrm{n}=2,130)$ and controls $(\mathrm{n}=3,975)$ from nine OCAC studies (Supplementary Table 1). Genotype data for all the previously reported TERT SNPs [9] has been excluded from the current analysis. Stepwise models were adjusted for study and age (at interview for controls and at diagnosis for cases), with a threshold of $P \leq 0.05$ for addition (forward stepwise) or removal (backward stepwise) of SNPs. All single marker risk estimates were obtained from unconditional logistic regression models adjusted for age (where available) and additionally for study where data was pooled across multiple studies. Assuming a log additive model of inheritance, the per-allele odds ratios (ORs) and their 95\% confidence intervals (CIs) associated for selected SNPs were estimated by fitting the number of rare alleles carried as a continuous covariate. Ovarian cancer risk associated with SNP genotypes were obtained for all invasive cases as well as a subset of serous cases. Breast cancer risk estimates were obtained for invasive cases and by estrogen receptor (ER) status. Separate comparisons were made for cases diagnosed before 50 vs. $\geq 50$ years of age to explore effect modification by advancing age of diagnosis. Summary estimates from pooled analyses using genotyped and imputed SNP data were obtained from weighted meta-analysis of study-specific parameter estimates ( $\beta$ coefficients and Standard Error). The minor allele frequency (MAF) for each SNP was estimated from the control population for each study. Study heterogeneity and risk differences associated with age groups ( $<50$ vs. $\geq 50)$ were assessed using the likelihood ratio test to compare logistic regression models with and without a multiplicative interaction term. All tests for association were twotailed, statistical significance was assessed at $P \leq 0.05$, and were performed in STATA SE v.11 (StataCorp, USA), and SAS v. 9.1. Tests for study heterogeneity and age group interaction tests were implemented in the $\mathrm{R}$ project for Statistical Computing (http:// www.r-project.org/).

\section{VEGAS applied to dbGAP data}

To evaluate evidence for association at TERT and CLPTM1L with various cancers, we applied the gene-based test implemented in VEGAS (all SNPs in gene test) [18] to data from dbGAP. In brief, we selected cancer cases and controls from dbGAP that were genotyped on $\sim 550,000$ SNPs (Illumina 610 quad or Illumina HumanHap550 arrays). Studies were CGEMS breast (1145 cases, 1142 controls), CGEMS pancreatic cancer (2328 cases, 2351 controls), GENEVA lung cancer (EAGLE and PLCO combined 2748 cases, 2840 controls) and CGEMS prostate (1145 cases, 1054 controls). For full details see dbGAP website (http://www.ncbi. nlm.nih.gov/gap). In each case, following standard quality control, the genomic control lambda was as would be expected if cases and controls were well matched; breast, pancreatic, lung, prostate lambda values $1.01,1.01,1.03,1.03$, respectively. The VEGAS gene based $P$ values from each of the four dbGAP studies were combined in a meta-analysis using Fisher's method for combining $P$ values.

\section{Luciferase assays}

Variants were introduced into pGL3-hTERT-3915 [15] by site-directed mutagenesis (Agilent Technologies). The A-A vector was generated by introducing the variants sequentially. Cells were transfected using siPORT $\mathcal{N} e o F X$ Transfection Agent (Ambion), according to the manufacturer's instructions, and harvested after $48 \mathrm{~h}$. Cells were washed with phosphate buffered saline (PBS) and lysed with $200 \mu \mathrm{L} /$ well lysis buffer $(25 \mathrm{mM}$ tris $\mathrm{pH} 7.8$, $2 \mathrm{mM}$ EDTA, 10\% glycerol, 1\% Triton X-100, $0.2 \mathrm{mM}$ DTT). Luciferase activity was assayed in triplicate for each transfection using $20 \mu \mathrm{L}$ lysate and $50 \mu \mathrm{L}$ reconstituted luciferase assay reagent (Promega). Luminescence was measured immediately for $4 \mathrm{~s}$ in each well with a Wallac Victor ${ }^{3} 1420$ multilabel counter. The experiment was repeated three times and the results averaged. Data were analysed by one-way ANOVA with post hoc Dunnett's tests in GraphPad Prism version 5.03 (GraphPad Software). 


\section{Supporting Information}

Table S1 Participating EOC case-control studies. (DOC)

Table S2 Per allele OR for all SNPs in EOC. (DOC)

Table S3 The association of rs2736109 with EOC, by study. (DOC)

Table S4 Participating invasive breast case-control studies. (DOC)

Table S5 The association of rs2736109/rs2736108 with risk of invasive breast cancer, by study.

(DOG)

Table S6 Breast cancer risk by age.

(DOC)

Table S7 Breast cancer risk by ER status.

(DOG)

\section{References}

1. Baird DM (2010) Variation at the TERT locus and predisposition for cancer. Expert Rev Mol Med 12: e16.

2. Rafnar T, Sulem P, Stacey SN, Geller F, Gudmundsson J, et al. (2009) Sequence variants at the TERT-CLPTM1L locus associate with many cancer types. Nat Genet 41: 221-227.

3. Shete S, Hosking FJ, Robertson LB, Dobbins SE, Sanson M, et al. (2009) Genome-wide association study identifies five susceptibility loci for glioma. Nat Genet 41: 899-904.

4. Landi MT, Chatterjee N, Yu K, Goldin LR, Goldstein AM, et al. (2009) A genome-wide association study of lung cancer identifies a region of chromosome 5p15 associated with risk for adenocarcinoma. Am J Hum Genet 85: 679-691.

5. McKay JD, Hung RJ, Gaborieau V, Boffetta P, Chabrier A, et al. (2008) Lung cancer susceptibility locus at 5p15.33. Nat Genet 40: 1404-1406.

6. Petersen GM, Amundadottir L, Fuchs CS, Kraft P, Stolzenberg-Solomon RZ, et al. (2010) A genome-wide association study identifies pancreatic cancer susceptibility loci on chromosomes 13q22.1, 1q32.1 and 5p15.33. Nat Genet 42: 224-228.

7. Turnbull C, Rapley EA, Seal S, Pernet D, Renwick A, et al. (2010) Variants near DMRT1, TERT and ATF7IP are associated with testicular germ cell cancer. Nat Genet 42: 604-607.

8. Rothman N, Garcia-Closas M, Chatterjee N, Malats N, Wu X, et al. (2010) A multi-stage genome-wide association study of bladder cancer identifies multiple susceptibility loci. Nat Genet 42: 978-984.

9. Johnatty SE, Beesley J, Chen X, Macgregor S, Duffy DL, et al. (2010) Evaluation of candidate stromal epithelial cross-talk genes identifies association between risk of serous ovarian cancer and TERT, a cancer susceptibility "hotspot". PLoS Genet 6: e1001016.

10. Ramus SJ, Vierkant RA, Johnatty SE, Pike MC, Van Den Berg DJ, et al. (2008) Consortium analysis of 7 candidate SNPs for ovarian cancer. Int J Cancer 123: $380-388$.

\section{Acknowledgments}

We are grateful to the family and friends of Kathryn Sladek Smith for their generous support of Ovarian Cancer Association Consortium through their donations to the Ovarian Cancer Research Fund. MARIE thanks Tracy Slanger and Elke Mutschelknauss for their valuable contributions, and S. Behrens, R. Birr, W. Busch, U. Eilber, B. Kaspereit, N. Knese, K. Smit, for their excellent technical assistance. GESBC thank Ursula Eilber and Tanya Koehler for competent technical assistance. HJO and HMO acknowledge the support of Matthias Dürst, Peter Schürmann and Peter Hillemanns. UKO thanks Ian Jacobs, Eva Wozniak, Andy Ryan, Jeremy Ford and Nayala Balogun for their contribution to the study.

\section{Author Contributions}

Conceived and designed the experiments: GG-T RRR ELG DNR. Performed the experiments: JB HAP XC MDS SA CB. Analyzed the data: JB HAP SEJ JL KM SM YL. Contributed reagents/materials/analysis tools: RTP DL ED SL IV JC-C SN AV DF-J MTG GL LRW RKM NB NA IBR TD BLF RAV ELG EVB SHO IO LR-R LEK NDL LSC AB-W IC SAG SJR AG-M UM PDP AMD SW-G UE KM AL AC SW DFE RRR GG-T LAK KKHA TM LFAGM. Wrote the paper: JB HAP GG-T.

11. Zhang B, Beeghly-Fadiel A, LongJ, Zheng W (2011) Genetic variants associated with breast-cancer risk: comprehensive research synopsis, meta-analysis, and epidemiological evidence. Lancet Oncol 12: 477-488.

12. Antoniou AC, Wang X, Fredericksen ZS, McGuffog L, Tarrell R, et al. (2010) A locus on 19p13 modifies risk of breast cancer in BRCA1 mutation carriers and is associated with hormone receptor-negative breast cancer in the general population. Nat Genet 42: 885-892.

13. Bolton KL, Tyrer J, Song H, Ramus SJ, Notaridou M, et al. (2010) Common variants at $19 \mathrm{p} 13$ are associated with susceptibility to ovarian cancer. Nat Genet 42: $880-884$.

14. Turnbull C, Ahmed S, Morrison J, Pernet D, Renwick A, et al. (2010) Genomewide association study identifies five new breast cancer susceptibility loci. Nat Genet 42: 504-507.

15. Chen YJ, Campbell HG, Wiles AK, Eccles MR, Reddel RR, et al. (2008) PAX8 regulates telomerase reverse transcriptase and telomerase RNA component in glioma. Cancer Res 68: 5724-5732.

16. Kyo S, Takakura M, Kanaya T, Zhuo W, Fujimoto K, et al. (1999) Estrogen activates telomerase. Cancer Res 59: 5917-5921.

17. Yi X, Shay JW, Wright WE (2001) Quantitation of telomerase components and hTERT mRNA splicing patterns in immortal human cells. Nucleic Acids Res 29: $4818-4825$.

18. Liu JZ, McRae AF, Nyholt DR, Medland SE, Wray NR, et al. (2010) A versatile gene-based test for genome-wide association studies. Am J Hum Genet 87: $139-145$.

19. Choi J, Southworth LK, Sarin KY, Venteicher AS, Ma W, et al. (2008) TERT promotes epithelial proliferation through transcriptional control of a Myc- and Wnt-related developmental program. PLoS Genet 4: e10.

20. Dunning AM, Healey CS, Baynes C, Maia AT, Scollen S, et al. (2009) Association of ESR1 gene tagging SNPs with breast cancer risk. Hum Mol Genet 18: 1131-1139. 